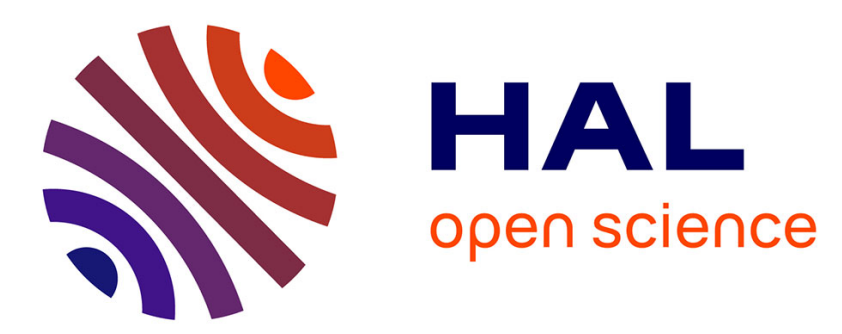

\title{
La collaboration festive: une éthique de fondation des lieux
}

\author{
Mathias Rollot, Chris Younès
}

\section{To cite this version:}

Mathias Rollot, Chris Younès. La collaboration festive: une éthique de fondation des lieux. Revue française d'éthique appliquée, 2019, No8 (2), pp.142. 10.3917/rfeap.008.0142 . hal-02494970

\section{HAL Id: hal-02494970 \\ https://hal.science/hal-02494970}

Submitted on 20 Nov 2020

HAL is a multi-disciplinary open access archive for the deposit and dissemination of scientific research documents, whether they are published or not. The documents may come from teaching and research institutions in France or abroad, or from public or private research centers.
L'archive ouverte pluridisciplinaire HAL, est destinée au dépôt et à la diffusion de documents scientifiques de niveau recherche, publiés ou non, émanant des établissements d'enseignement et de recherche français ou étrangers, des laboratoires publics ou privés. 


\section{La collaboration festive: une éthique de fondation des lieux}

MATHIAS ROLLOT

ARCHITECTE ET CHERCHEUR EN ARCHITECTURE, UMR AUSSER 3329, ENSEIGNANT CONTRACTUEL À L'ÉCOLE D'ARCHITECTURE DE MARNE-LA-VALLÉE

CHRIS YOUNĖS

PSYCHOSOCIOLOGUE, DOCTEURE ET HDR EN PHILOSOPHIE, PROFESSEURE À L'ÉCOLE SPÉCIALE D'ARCHITECTURE

La contribution voudrait mettre en lumière dans quelle mesure enjeux et éthiques sont à l'œuvre au sein des pratiques synergiques, en se concentrant plus particulièrement sur la figure de la collaboration dans les « collectifs » d'architectes. Comment et en quoi la collaboration, que ces derniers placent au cour du processus de projet, participe-t-elle d'une reprise des manières d'un faire et d'un être ensemble au monde?

\section{Co-labeur, convivialité et conflits}

Le «labeur» partagé de la fondation des lieux a de tous temps été associé à des démarches symboliques, des codes et des rituels festifs autant qu'à des conflits pouvant être d'une violence extrême. Ainsi, René Girard a exploré une anthropologie fondamentale selon laquelle toute société humaine est fondée sur une violence qui est inscrite en son cœur et se trouve transmuée dans l'ordre du sacré, que ce soit dans des rites archaïques, païens, dionysiaques ou dans les rites judéo-chrétiens (Girard, 1972 ; 1978). De même, Mircea Eliade a bien montré dans son étude historique Le sacré et le profane à quel point toute installation territoriale passe par des pratiques ritualisées destinées à fonder collec-

1. «Friedrich Rückert considérait que le centre du monde était l'Allemagne, le centre de l'Allemagne la Franconie, le centre de la Franconie Schweinfurt, le centre de Schweinfurt sa maison et le centre de sa maison le cœur de sa bienaimée. II arrive que l'omphalos soit privé. » (Westphal, 2011, p. 39) tivement les lieux autour d'un imaginaire rendant la terre nue habitable. "On ne fait "sien" un territoire qu'en le "créant" de nouveau» (Eliade, I957, p.34), nous dit Eliade de sorte qu'il n'est nullement surprenant que "même dans les sociétés modernes, si fortement désacralisées, les fêtes et les réjouissances qui accompagnent l'installation dans une nouvelle demeure gardent encore le souvenir des festivités bruyantes qui marquaient jadis l'incipit vita nova» (ibid., p. 55). C'est ainsi que tout omphalos («ombilic»: centre et origine cosmogonique du monde), si mineur, temporaire, voire privé soit-il ${ }^{1}$, est nécessairement le fruit d’un 
processus de fondation symbolique, partagé et intime à la fois. C'est-àdire que toute fondation topique naît de l'établissement premier d'un omphalos partagé et partageable. Ailleurs et autrement encore, Michel Maffesoli a lui aussi exploré, dans bon nombre de ses travaux, les multiples formes et forces à l'œuvre dans les dynamiques festives, y reconnaissant une santé populaire et la possibilité de réguler les violences accumulées par les conflits de valeurs et d'intérêts. Toutes ces analyses concourent à la même conclusion : c'est paradoxalement la violence de la festivité (émotionnelle, symbolique, sociale) qui ouvre les pratiques festives à des capacités transformatrices pour l'existence; par là, ces dernières peuvent moduler la vie collective en engageant une commune énergie affective, et renouer avec les forces vives et excessives que la figure de Dionysos a représentées, en contradiction et complémentarité avec la figure apollinienne de la raison apaisée.

Pure liminalité, la festivité créatrice de topos est elle aussi acte d'échange en ce qu'elle n'appartient ni à l'ordre de l'habité et ni à celui du non-habité, mais réussit justement la prouesse de se tenir entre ces deux domaines existentiels. Renversant cette dualité pour établir temporairement un topos poïétique, elle forme un omphalos qui serait à la fois promesse d'un futur habitable et révocation des violences sociétales passées. On pourrait dès lors rapprocher aussi la festivité créatrice du topos de l'échange symbolique chez Jean Baudrillard - ce « rapport social qui met fin au réel, qui résout le réel, et du même coup l'opposition entre le réel et l'imaginaire» (Baudrillard, I976, p.205). La transformation des géographies naturelles en lieux culturels transcende les catégories dialectiques de l'habitable et de l'inhabitable, de l'intériorité et de l'extériorité, de l'individuel et du collectif ou encore de l'arbitraire et du rationnel pour s'établir en termes plus dialogiques et dynamiques à la fois (Rollot, 20I7). Milieu au sens aussi de l'entre, elle est béance, passage, métamorphose.

De Jan Sapp à Lynn Margulis et jusqu'à Pierre Kropotkine, nombreux sont les travaux scientifiques qui ont pu donner à lire l'importance des symbioses, synergies et processus collaboratifs pour le monde du vivant: humains et non-humains (Sapp, I994; Margulis, I99I ; Kropotkine, I904). Plus récemment, c'est Richard Sennett, dans Ensemble pour une éthique de la coopération, qui considéra à son tour l'importance du besoin humain de coopération, et mit l'accent sur ce «fondement du développement humain», autant que sur l'importance du savoir «faire avec» (Sennett, 20I4). L'enjeu, pour le penseur, est de se situer dans l'expérience ordinaire tout en réapprenant à considérer celle-ci, par le biais notamment de la «conversation dialogique»: un échange composé à la fois par l'empathie et la capacité d'engagement des parties autant que par la mise en place de rituels propices à des ambiances d'écoute, d'invention et de négociation. Cette éthique de la discussion est pour lui condition de possibilité à la production de nouvelles synergies coopératrices capables de soutenir des projets proposant des perspectives viables, vivables et équitables. Concrètement, cela signifie s'adapter aux évolutions des pratiques, promouvoir des valeurs de l'agir passant d'une culture du top-down, qui a démontré son insuffisance, à une culture du bottom-up associée à des partenariats horizontaux : autant de voies à même de renforcer les marges de 
manœuvre des habitants dont Michel de Certeau, dans son exploration de L'invention du quotidien, avait bien décelé l'importance fondamentale pour l'habitation humaine (Certeau, I980).

La collaboration est une occasion de penser les processus des projets sociaux et spatiaux dans leurs réalités complexes. Collaborer, en ce sens, c'est envisager le chantier, ses phases, matières et métabolismes, ses potentialités sociales, politiques et esthétiques. Collaborer, c'est considérer pleinement la diversité des protagonistes de la fabrique et de la vitalité urbaine, ainsi que leurs intérêts, pouvoirs, désirs et intérêts propres. Collaborer, c'est se tourner vers les ressourcements effectifs synergétiques (vivant, écosystémique, climatique ou géographique) latents en chaque échelle et entre les échelles. Collaborer, c'est entrevoir en l'Autre et son altérité radicale la possibilité de se redécouvrir soi-même : un processus toujours renouvelé et renouvelable à toutes les phases du projet, et avec tous les co-existants humains et non humains impliqués par lui. Collaborer, c'est faire de la place au mineur, au singulier et à l'étrange face à la domination, à l'uniformité et à la destructivité vers laquelle tendent les sociétés capitalistes ; c'est lutter contre les sexismes, racismes, classismes et spécismes courants et contre la fabrication matérielle de la ville conformiste.

Or, ces éthiques de la fondation des lieux par la festivité, de la collaboration créatrice ou de l'a priori éthique du «faire ensemble» comme valeur en soi, sont justement les directions parallèles qu'ont pu suivre bon nombre de contre-cultures architecturales de ces dernières années, par-delà les dualités limitatives du sachant et du non-sachant, du plan et de l'œuvre, de la commande et de son exécution par un tiers. Par elles, il n'est nullement question dans l'idée de collaboration d'une quelconque délégation, pour autant que «faire avec», n’a jamais signifié «faire faire par les autres », et en aucune façon la fabrication collective des établissements humains ne doit être confondue avec une dépossession des compétences, rôles et responsabilités architecturales. Tout au contraire, c'est d'une reprise disciplinaire créatrice par « extension du domaine de l'architecture » dont il est question.

\section{"Collectifs " et faire ensemble}

Depuis deux décennies, des "collectifs d'architectes» réinventent certains processus de la fabrication urbaine de notre époque anthropocène en souffrance et actualisent, très concrètement, les belles intentions ici énoncées. Ces collectifs, en recourant aux processus collaboratifs, réinstaurent des stratégies et tactiques de construction de la ville par le biais de fondations ontologiques, imaginaires, symboliques et éthiques mieux partagées que celles, purement économiques et matérielles, de la modernité capitaliste. Il n'est nul besoin de former un «collectif» pour « collaborer» ou pour refuser ces constats de mortifications des processus urbains. S'il importe pourtant de s'arrêter sur ces formations, c'est en ce qu'elles sont l'émergence visible d'une promesse plus large, les extraordinaires promoteurs d'une société nouvelle d'une promesse plus large, bref: un moyen et non une fin en soi. 
Parmi un certain nombre de publications récentes spécifiquement sur ce sujet (Macaire, 20I2 ; Bianchetti, 2015 ; Gatta, 20I8), relevons des ouvrages clés, qui ont prospecté ces modifications qui renouvellent les métiers de l'architecture; deux directions d'ouvrages à même d'éclaircir ces postures éthiques nouvelles et les réalisations concrètes par le biais desquelles elles trouvent à s'actualiser; et deux ouvrages réalisés dans le cadre de Biennales d'architecture de Venise ( $13^{\mathrm{e}}$ et ${ }^{6} 6^{\mathrm{e}}$ éditions), qui ont donné lieu à des débats publics nationaux sur le sujet.

En 20I2, tout d'abord, AlterArchitectures Manifesto (Paquot et coll., 20I2) a investigué en précurseur ces nouvelles conditions de production de l'architecture, de l'urbain et du paysage. Une constellation de projets réalisés dans différentes villes européennes est dessinée par le livre, témoignant d'échanges et collaborations entre décideurs, habitants et professionnels. Ces projets, qui ont tous contribué à l'émergence de multiples micro-initiatives citoyennes, y étaient perçus comme la possibilité d'envisager d'autres processus de constructions de biens communs pour nos villes. Comme le synthétise bien Édith Hallauer, il est question «de seconds chantiers, de mutabilité urbaine, d'architecture ordinaire, de recouvrement des communaux, d'incrémentalisme, de ville par le vide, de la décroissance comme projet urbain, des arts du territoire, d'architecture pirate, du devoir de ville et d'urbanisme thermodynamique» (Hallauer, 2015).

Plus récemment, L'hypothèse collaborative. Conversation avec les collectifs français d'architectes (ateliergeorges et Rollot, 20I8) a rendu compte de la vitalité de telles initiatives sur le territoire français. Fruit d'une enquête menée entre 2015 et 2018 par le biais d'entretiens avec les collectifs eux-mêmes, l'édition propose une confrontation de ces paroles avec des points de vue et analyses de chercheurs, de maîtrises d'ouvrages et d'architectes en contact avec ce milieu. Ce livre fut l'occasion de mises en débat publiques dans de hauts lieux des réseaux architecturaux français: au sein du Pavillon français de la Biennale d'architecture de Venise en mai 2018 (six heures de débats in situ retransmis à la radio) et du Pavillon de l'Arsenal de Paris en octobre 20 I8 (trois heures sous le même format). Ces débats, tout comme l'ouvrage lui-même, ont eu pour vocation et résultat de mettre en lumière la grande complexité de cette appellation « collectifs» en architecture, en faisant clairement apparaître la grande diversité au sein de cette famille mouvante, parfois inassumée par ses membres même, voire à l'occasion conflictuelle-les rassemblements nationaux comme Superville étant tantôt de joyeux échanges et retrouvailles, mais aussi, d'autres fois, l'occasion de débats et de vives oppositions sur le sens, les enjeux et directions éthiques et politiques à donner à ces dynamiques «collectives» en cours. Ils sont la preuve même que «les collectifs» n'ont encore rien, pour l'heure, d'une grande famille soudée ou d'un unique milieu aux contours bien défini. Leur programme politique autant que leurs outils varient grandement d'un protagoniste à l'autre : d'où la nécessité de ces ouvrages analytiques, pour penser ces émergences aux dynamiques et contenus complexes.

Disons en synthèse à quel point ces ouvrages invitent tous deux à considérer avec prudence ce label de «collectif», qui plus est à l'heure où ce dernier apparaît de plus en plus fréquemment détourné, voire 
2. Sur les apports de Berque et Simondon à cette éthique des milieux, voir notamment Duhem (2018) récupéré, par certains rouages de la société à laquelle il prétendait échapper. À mesure que sortent les commandes, concours et autres appels à projets incluant d'emblée la présence souhaitée d'un «collectif » pour participer à la valorisation foncière d'un projet immobilier, c'est le projet collaboratif lui-même qui perd en force de détournement et de réinvention; c'est la marge, ses interstices et fissures encore habitables qui se réduisent sous le poids des majors du BTP, de la société technocratique et de la ville néo libérale.

\section{Vers des dispositifs coopératifs émancipateurs}

Manière de faire souple, adaptable, inventive et vivifiante dès lors qu'elle est déployée sur des bases festives, l'éthique de la collaboration est une forme "d'impératif catégorique de processus» qui contient intrinsèquement tous les éléments d'une «éthique des milieux » (Younès et Paquot, 2000; Younès et Goetz, 2010²). Certes, cette modalité d'action ne peut s'établir que sur la base de vertus précieuses - quelque ouverture d'esprit, quelque empathie ou tempérance. Et, de même, il faut bien reconnaître que ses conséquences concrètes peuvent bien s'avérer fluctuantes (principe même d'une philosophie de l'ouvert, de l'imprévu et du dialogue), à savoir que ce n'est pas parce que collaboration il y a que résultat enthousiasmant il y aura. Jean-François Lyotard écrit que le "peuple» post-moderne "diffère du tout au tout de celui qui est impliqué dans les savoirs narratifs traditionnels, lesquels, on l'a dit, ne requièrent nulle délibération instituante, nulle progression cumulative, nulle prétention à l'universalité ». Pour le philosophe, c'est en cela précisément qu'«il n'y a pas à s'étonner que les représentants de la nouvelle légitimation par le "peuple" soient aussi des destructeurs actifs des savoirs traditionnels des peuples, perçus désormais comme des minorités ou des séparatismes potentiels dont le destin ne peut être qu'obscurantiste » (Lyotard, I979, p.52-53)...

Si cela est entendu, comment nier toutefois la valeur intrinsèquement éthique du processus collaboratif - fût-ce dans une société post-moderne capable de simulacres de concertation déconcertants? Critique sociétale, modèle antipaternaliste, la collaboration est un outil de tout premier choix pour lutter contre la machine technocratique et ses rouages toujours plus tortueux, empoisonnants et dévitalisants pour la construction de l'être. Processus convivial, elle forme un dispositif efficace pour lutter contre le phénomène toujours plus pressant des bullshit jobs (Graeber, 2018) et la multiplication des burn out, bore out et brown out - un phénomène aux conséquences sanitaires aussi incalculables que démesurées.

Collaborer, au sein du système technocratique actuel, ne saurait être une visée innocente. Déployée comme acte de résistance, la collaboration est à la fois éthique appliquée et valeur morale. C'est une première amorce vers une société biorégionale, frugale et vivifiante, qui reste encore à inventer (Rollot, 20I8): un formidable outil de réinvention des possibles à l'heure où des témoignages professionnels conduisent à considérer que la transition écologique requiert moins de montrer ce qui se perd que d'imaginer d'autres possibles. Ensemble à l'ouvrage: 
quand coopérer fait habiter (Blanc, 20I7), c'est ainsi ce que préconise Bernard Blanc, directeur d'Aquitanis (organisme HLM). Au travers d'un livre éponyme, sont présentés les récits multifacettes d'une écologie du faire ensemble en architecture afin de redonner droit de cité à l'horizon d'un sens partagé. Une orientation similaire est poursuivie par Dominique Gauzin-Müller dans le collectif Habitat social d'aujourd'hui (2018) qui décrit quarante opérations dans des villages ou des métropoles voulant prendre soin des vulnérabilités et trouver d'autres formes d'hospitalité, d'économies de moyens, de matières et d'énergies, de recyclage et de convivialité.

\section{Enseigner la collaboration ? \\ Compatibilités et résistances en école d'architecture}

L'enseignement du projet architectural, urbain, paysager, pourrait-il, devrait-il, dès lors, devenir collaboratif et festif à la fois? La question est d'une actualité brûlante, et ne fait pas l'unanimité au sein des écoles d'architecture françaises. il faut dire que ces formes projectuelles plus processuelles, inclusives et ouvertes à l'imprévu entrent en opposition, a priori, avec un certain nombre de caractéristiques «classiques» de la profession architecturale : le plan et sa nécessaire «planification» figée, la figure de l'expert ou encore les responsabilités pénales d'un métier qui toutes nécessitent, a priori, de minimiser les inconnus et parts d'incertitudes du projet. De même, ce sont dans leurs fondements que sont interrogées nos écoles d'architecture plus traditionnellement portées sur le « projet » lui-même (ses formes et ses fonctions, ses caractères de stabilité, d'utilité et de beautés, sa composition ou ses références disciplinaires internes) que sur les processus d'actualisation qui pourraient le porter vers le réel (filières, acteurs, économies, chantiers, normes, situations écologiques particulières, etc.). La réticence d'une arrière-garde conservatrice d'architectes modernes est grande, dans les écoles, au sujet de ces mouvements collaboratifs compris comme des mouvements faisant perdre de vue aux étudiants le projet d'architecture lui-même. Comment, dans ce contexte, le projet collaboratif pourrait-il bien être «enseigné »? Il faut bien expliciter le déplacement des critères d'évaluation du projet sous-tendu par ces changements - la migration de critères très disciplinaires vers une évaluation morale plus large vis-à-vis d'une société et des anthropo-écosystèmes auxquels elle contribue-, pour que soient mis en lumière tant la raison d'être que le bien-fondé moral de ces initiatives radicalement transformatrices pour l'architecture, l'urbanisme et le paysage.

Parmi les initiatives favorables à la proposition, relevons notamment la tenue de récentes journées d'étude ${ }^{4}$, quelques rencontres internationales ${ }^{5}$, ou, plus spécifiquement encore, la tenue tout actuelle du " séminaire de formation continue en pédagogie » organisé par le réseau Pédagogie architecture arts et paysage (PAPier) les 28 et 29 mars 2019, qui portait cette année sur le thème de «La coopération-collaboration : l'apprendre, l'enseigner, l'évaluer». Le texte introductif au séminaire pose clairement les opportunités en jeu dans la collaboration :
3. Référence à la célèbre trinité vitruvienne firmitatis, utilitatis, venustatis. Cf. Vitruve, De l'architecture, -23 av. J.C. Sur les multiples traductions faites de ce texte, voir B. Queysanne, " Les mots et la discipline. Sur la traduction de quelques propositions vitruviennes ", Cahiers thématiques de Lille, MSH, $\mathrm{n}^{\circ} 1$, 2001, p. 68-81.

4. Dont notamment «Écrire pour/écrire avec : interroger les processus et espaces participatifs ", Journée d'étude organisée par le Syndicat Potentiel, Barbara Morovich et coll., Strasbourg, 5 décembre 2018.

5. Comme l'événement "Designing Community ", organisée par Noödesign et l'EHESS à l'Espace Niemeyer Paris, avril 2019. 
«Utiliser la coopération-collaboration rompt avec les pratiques pédagogiques traditionnelles et redéfinit les rôles des enseignants tout autant que ceux des étudiants. Le savoir y passe moins par l'expert transmetteur et plus par une mise en avant-plan des capacités de réflexion, de responsabilisation et d'organisation des étudiants. Elle fait place à l'entraide, à la solidarité, à l'ouverture aux autres, aux compromis, au droit à l'erreur, mais aussi à la confiance mutuelle et au plaisir d'apprendre. Au cœur de cette façon de travailler ensemble à atteindre un objectif commun, on retrouve aussi l'interdépendance positive entre les membres du groupe: comme les maillons d'une chaîne, chacun est essentiel au bon fonctionnement, à la réussite et à l'apprentissage. Chaque membre est perçu comme une ressource, y apporte ses forces, ses connaissances et il bénéficie de celles des autres. Les complémentarités sont exploitées par les interactions et la négociation.»

C'est que l'enseignement de la collaboration (comme fin, comme contenu), en effet, n'est pas envisageable sans un enseignement lui-même collaboratif (en tant que moyen). Et que, loin d'un processus codifié ou précisément défini, cet enseignement est envisageable au moyen d'une multitude de modalités: comme cela a été dit déjà, la collaboration ne doit jamais être réduite ou confondue avec les seuls processus "participatifs» et "concertatifs», ou les formes juridiques ou pratiques du «collectif d'architectes ». Face à l'omniprésence historique de l'objet au sein de la discipline architecturale, l'enjeu est celui d'une reconnaissance de l'importance tout aussi fondamentale de bâtir des "projets-processus» (de conception, d'actualisation, de durée de vie et de mort), qui travaillent ces objets bâtis. Une fois que peut être reconnue la pertinence de ces formats de conception plus dynamiques (et non moins pragmatiques, sérieux, engagés et responsables), c'est une grande part des difficultés et paradoxes pédagogiques qui s'effacent. À savoir que la pédagogie du projet architectural collaboratif peut s'appuyer sur une pensée et une valorisation tant de l'objet que des processus architecturaux :

- En se concentrant sur les conditions de possibilité du dialogue avec la société civile ou le non-humain, le projet d'architecture, d'urbanisme ou de paysage peut travailler à déployer des espaces, dessins, perspectives et discours au service d'un processus de dialogue pleinement partagé et équitable avec des groupes non experts et leurs diversités d'origines, de points de vue et d'intérêts.

- En s'appuyant sur les principes fondateurs de laisser-faire, de tiers-paysage, ou encore de jardin en mouvement du paysagiste-jardinier Gilles Clément, s'ouvre la possibilité d'une conception qui aille dans le sens d'une «déprise d'œuvre» (la formule est d'Édith Hallauer, 20I7): un projet capable de travailler avec l'ouvert et l'inconnu, et de tenir des positions sans fermer les possibles, pour l'humain comme pour le non-humain.

- En travaillant les relations, reliances, hiérarchies et structures du rhizome collaboratif plutôt qu'en s'accrochant désespérément aux résultats qui pourraient en naître, leurs formes et fonctions, les projets d'architecture, d'urbanisme et de paysage pourraient contribuer à 
inventer des formes de «synergies urbaines» (D'Arienzo et Younès, 20I8) écologiques mieux capables de contribuer aux fonctionnements écosystémiques des établissements humains.

- En se concentrant sur les différences entre probable et possible, et les horizons de la «narration/fabulation spéculative» théorisés par Donna Haraway ${ }^{6}$, tout autant qu'en s'appuyant sur les narrations créatives de Luca Merlini (20I0 ; 20I7), le projet peut repenser ses relations au récit, à la fiction, à la prospective et à la prévision (ensemble formant tout de même une part non négligeable des attentes enchaînées à cette profession à qui est demandé un certain nombre de " garanties »).

- En s'ouvrant aux écrits d'auteurs éco-féministes tels que Starhawk (2015), le projet d'architecture pourrait nourrir son champ de références de tout un ensemble de rituels, de stratégies et tactiques critiques et conviviales, de dispositifs de fondations énergétiques capable de dynamiser les processus de collaboration et de conception à la fois, au service de luttes progressistes épanouissantes pour les individus.

Comment les parties prenantes des projets pourraient-elles être indifférentes à pareilles métamorphoses vivifiantes? L'art de liens retrouvés et féconds, grâce à des dispositifs ouverts exprime des transitions décisives dans lesquelles les passages effectués sont d'ordre éthique, esthétique et politique. Par la collaboration entre les divers protagonistes de l'instauration des lieux d'habiter ou de l'enseignement, sont repris le sens de l'action commune et celui de la responsabilité. Dialoguer, prendre la parole, co-imaginer, co-concevoir, coréaliser, tout cela construit un écosystème transculturel d'un autre type, à même de contribuer à susciter d'autres formes d'accord pour développer d'autres en-commun.

\section{Bibliographie}

ATELIERGEORGES ; RoLLOT, M. 2018. L'hypothèse collaborative. Conversation avec les collectifs français d'architectes, Marseille, Hyperville.

BAUDRILLARD, J. 1976. L'échange symbolique et la mort, Paris, Gallimard.

BIANCHETTI, C. (sous la direction de). 2015. Territoires partagés. Une nouvelle ville, Genève, MétisPresse.

BLANC, B. 2017. Ensemble à l'ouvrage. Quand coopérer fait habiter, préface C. Younès, Plaissan, Museo.
6. Notons le brillant documentaire de Fabrizio

Terranova sur Donna Haraway intitulé Donna Haraway: Story telling for Earthly Survival, réalisé en 2016 et depuis en tournée permanente à l'international, https:// earthlysurvival.org
Certeau, M. (de). 1980. L'invention du quotidien, tome 1 : Arts de faire, Paris, Gallimard.

D'ARIENZO, R. ; YOUNĖS, C. (sous la direction de). 2018. Synergies urbaines. Pour un métabolisme collectif des villes, Genève, Métispresses.

DUHEM, L. 2018. "Prendre soin des êtres comme des choses. Réflexions éthiques pour un design des milieux de vie ", Revue française d'éthique appliquée, Toulouse, érès, p.125-132.

ELIADE, M. 1957. Le sacré et le profane, Paris, Gallimard. 
GATTA, F. 2018. (Contre)pouvoirs urbains, Paris, Donner Lieu.

GAUZIN-MULLER, D. 2018. Habitat social

d'aujourd'hui, préface C. Younès, Plaissan, Museo.

GIRARD, R. 1972. La violence et le sacré, Paris, Grasset.

GIRARD, R. 1978. Des choses cachées depuis la fondation du monde, Paris, Grasset.

GraEBER, D. 2018. Bullshit jobs, Paris, Les liens qui libèrent.

HALlaUeR, E. 2015. «Alterarchitecture Manifesto », hyperville.fr, 15 octobre.

HALlAUER, E. 2017. Du vernaculaire à la déprise d'œuvre : urbanisme, architecture, design, thèse de doctorat sous la direction de Thierry Paquot à l'Université Paris-Est.

KROPOTKINE, P. 1904. L'Entraide, un facteur d'évolution, Paris, Hachette.

LYOTARD, J.F. 1979. La condition postmoderne. Rapport sur le savoir, Paris, Les Éditions de Minuit.

MACAIRE, E. 2012. "L'architecture à l'épreuve de nouvelles pratiques : recompositions professionnelles et démocratisation culturelle ", thèse de doctorat sous la direction de Jodelle Zetlaoui-Léger à l'Université Paris Est.

MARGULIS, L. 1991. Symbiosis as a Source of Evolutionary Innovation: Speciation and Morphogenesis, Cambridge, MIT Press.
MERLINI, L. 2010. Le pays des maisons longues, Genève, Métispresses.

MerLINI, L. 2017. Le XIO. Dits et dessins

d'architecture, Genève, Métispresses.

PAQUOT, T. ; MASSON-ZANUSSI, Y. ; StATHOPOULOS, M. (sous la direction de). 2012. AlterArchitectures Manifesto, Gollion, Infolio.

Rollot, M. 2017. Critique de l'habitabilité, Paris, Libre et Solidaire.

Rollot, M. 2018. Les territoires du vivant. Un manifeste biorégionaliste, Paris, François Bourin.

SAPP, J. 1994. Evolution by Association: A History of Symbiosis, Oxford University Press.

Sennet, R. 2014. Ensemble. Pour une éthique de la coopération, trad. P-E. Dauzat, Paris, Albin Michel.

STARHAWK. 2015. Rêver l'obscur. Femmes, magie et politique, Paris, Cambourakis.

Westphal, B. 2011. Le monde plausible. Espace, lieu, carte, Paris, Les Éditions de Minuit.

YOUNÈS C. ; GOETZ, B. 2010. « L'architecture des Milieux », Le portique, $n^{\circ} 25$.

YounÈs, C. ; PAQuOT, T. (sous la direction de). 2000. Éthique, architecture, urbain, Paris, La Découverte. 